\title{
Multi-level QR code with Hill Climbed Encrypted Public Level for Secured Message Transfer
}

\author{
K Gokul, Ritu Garg
}

\begin{abstract}
With the world turning into a worldwide town, the data sharing requires abnormal state security with substantial verification of the data which ought not be undermined with the time, hence providing food all the above necessities we propose another brisk reaction $(Q R)$ code containing two stockpiling levels specifically open and private stockpiling levels, which can go above and beyond in validating the inventiveness of the reports. The open stockpiling level can be perused by any traditional $Q R$ code scanners while it's not the equivalent for the private stockpiling level as its being produced with exchanging the dark modules with finished examples of the first $Q R$ code consequently it tends to be perused by just explicit QR scanners. The data's being put away in the private stockpiling level is being encoded sing q-ary code substituted by mistake amendment systems along these lines expanding the QR stockpiling limit while the open stockpiling level data's are being scrambled with the key which will be sent to the private stockpiling level where it experiences all the procedure like the private data's and will be moved by means of QR to the required gatherings. The size can be expanded by the information's or with the finished example estimate.

Index Terms: QR code, multiple Storage levels, Secured transfer, Extended hill climbing algorithm.
\end{abstract}

\section{INTRODUCTION}

Today innovation being at its pinnacle the use of graphical codes is being unavoidable particularly codes like scanner tag, Quick Response (QR) code, Data Matrix are utilized routinely in different areas. Huge number of utilizations are being fabricate dependent on these codes, for example, promoting, redirection to sites, ticket and transport track and follow, distinguishing proof like general store items and so forth.

QR code is machine affirmation code organized and made by the Japanese Denso Wave Company, this has as of now seen by general specific by ISO and as a result of its low visual affirmation characteristics, getting the message from $\mathrm{QR}$ code by human vision is nearby immense. The transmission of characterized and moreover tricky data over the correspondence channels is so far being considered as a testing task. The prominence of the above codes depends on the accompanying qualities resembling heartiness, the productivity in perusing by any gadgets and by any client who sweeps the QR code with their high encoding limit with ran mistake redress procedures likewise the extra highlights like its size, accessibility and its heartiness to its geometric mutilations.

Revised Manuscript Received on July 16, 2019

Gokul K, M.tech Cyber security, National Institue of Technology, Kurukshetra, India

Dr. Ritu Garg, Computer Engineering, National Institue of Technology, Kurukshetra, India

Anyway supplementing, these attributes likewise prompts a portion of its downsides like

Availability of the data's encoded in QR code by every one of the clients who sweeps it considerably whether the data's are being figured or not or its being gotten to by genuine client or not. In this way to lessen traps and making the QR code with more proficiency in limit and security, Thus we propose the new thought wherein the current QR code with dark modules is being supplanted by explicit finished examples. With the presentation of new finished examples isn't just centered around the capacity limit yet in addition intended for the affectability to contortions dependent on the $\mathrm{P} \& \mathrm{~S}$ process. The finished example is basically founded on the private data handling subsequently it won't intrude on the standard perusing process which will be checked and perused by the typical scanner as a dark module notwithstanding when the finished examples is being mutilated or duplicated in any structures or the different in this way making the open data in its scrambled structure accessible all through. Subsequently included with polarization computerized holography with the QR code henceforth making it with developed key space with still progressively upgraded the security level of encryption system

The proposed QR code consists of two levels wherein which first level information may have studied by Normal QR code reader making it satisfying the characteristics of the normal QR code while the double level has being encoded using $q-$ ary $(q \geq 2)$ code with error correction capacities. Thus the data is hidden to the default QR code reader as it identifies the textured patterns with black modules thus making the double level useful in secret data sharing. Moreover with textured pattern being sensitive to $P \& S$ distortions hence the double level will be helpful in identifying the original Two Level QR code from their replicas and can be distinguished either.

\section{RELATED WORK}

The QR code [13] organized on two-dimensional code for putting away the data by the assistance of modules with two contracting hues. It comprises 40 variants dependent on different information limits. The first Version is made by $21 \times$ 21 modules. In this way following models raised with each four progressive modules on either headings, up to 40 adaptations, there by organized $177 \times 177$ modules. 


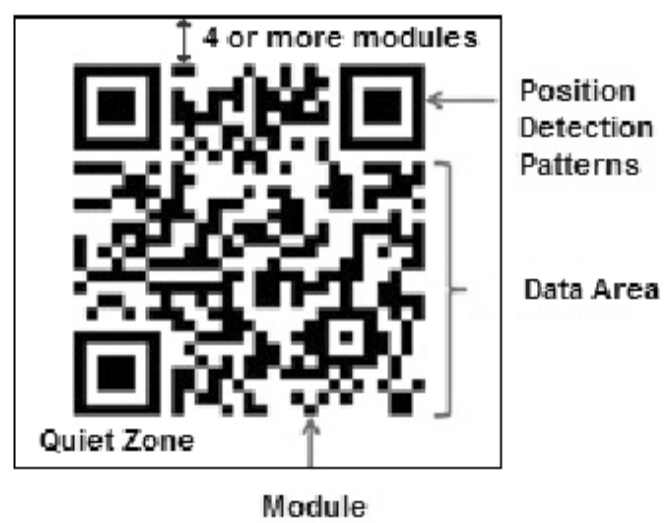

Fig 1: Structure of QR code

The QR comprises of to a great extent two sections, specifically encode region and different forms like the standard QR code parts. QR code contains radical highlights like many utilized, for example, geometric relationship with proficient deciphering attributes. To get the ideal structure position labels are being utilized to their ideal arrangement. The numerous arrangement designs which are for the most part utilized in mistake and disfigurement redress can be included for in excess of 2 variant number of the QR codes. Different highlights like mistake rectification code utilized when the code is harmed or torn are principally present in the rendition data zone.

With the escalated research [5] in QR code results that if cell with $3 \times 3$ modules could be supplanted, the exactness of perusing the $\mathrm{QR}$ code will increment radically.

With tremendous picking up in ubiquity for $\mathrm{QR}$ code so similarly as its being assaulted [5,6] is likewise on a high note to amass in QR code confirmation in a full encryption $[9,10]$ a printing procedure [7], brilliant examining [8],. Or maybe [11 12] acquire extra costs, encryption [9, 10] needs overwhelming computational time, however [9, 12] isn't being good by a standard decoder.

Wu and Liu [1] thought of a plan to utilize parallel pictures and inserting the information in ti dependent on the square based relationship However the picture twists is gigantic in this impact along these lines to beat this downside Yang and Kot [2] proposed the idea of visually impaired information with huge capacity limit and decent visual morals quality anyway this could be appropriate just to the paired components Varna et al. [3] proposed another methodology for drubbing information into the content reports with changing long arrangements of characters however this idea can be just relevant to the characters not ASCII esteems. Das et al. [4] thought of the thought by changing the other bend parameters in non-straight portions with the predefined shapes also.

Or maybe because of colossal mutilations in the double information and pictures the productivity is constrained with the decidability of the QR code is lessened. In this manner, another procedure plan is required for $\mathrm{QR}$ code.

\section{PROPOSED WORK}

The proposed QR code will be discussed below based on its categories like properties, construction, application, authentication in the end the demonstration of the proposed $\mathrm{QR}$ code. The section 3.A describes about the generation followed by the capacity and the subsequent section 3.B analyses about the application and authentication finally the section 3.C will be discussed about the experimental analysis of the proposed QR code

\section{A.Generations}

As talked about before The proposed twofold level QR code is like the current Standardized QR level codes as the customary QR codes incorporates arrangement designs, position labels, rendition configuration examples and timing designs though the real distinction differentiates the current one is the consideration of dark modules by finished modules and white modules. Anyway the substitution of dark modules with the surface examples ones does not change the typical QR code process, besides the consideration likewise builds the blunder remedy and capacity limit too.

The proposed staggered QR code is basically focuses on the validation and mystery message move and the plan by above both is the comparative with the little changes in the position region. In mystery data idea, the dark modules are traded with finished structures. Though, in the verification procedure, the position labels are not adjusted.

In the private message idea as said the substitution of the position labels is for the most part dependent on the change procedure which is for the most part utilized for the example identification and the above must be taken consideration in the interpreting procedure of the mystery message as this plays the base for the mystery message sharing situation. Dissimilar to the over the archive verification technique, The finished examples job is to contrast and the first examples of the first reports. Because of the above reason, the dark modules are not being changed.The public message(Mpub) that needs to be encrypted will undergo the encryption techniques while key that will be encrypted will also be sent via the QR code. The encryption technique[4] to be utilized here to encode the open key is the all-encompassing slope figure calculation where in which the accompanying advances will be tolerated to make the information scrambled. At first the open information which should be scrambled is taken and there is no imperative on the information both the tally and the information will be masterminded in the request of the square lattice where in which the request is being chosen by the framework and should likewise be possible by the client.

The above advance is trailed by the transformation of the information into its ASCII esteems with the goal that it will be helpful for the basic computational tasks. Also, the arranged square lattice will be numbered beginning from left and will be noted by the framework as needs be.

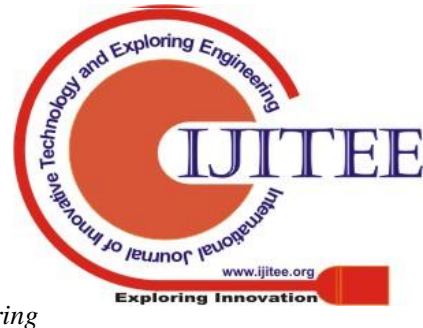




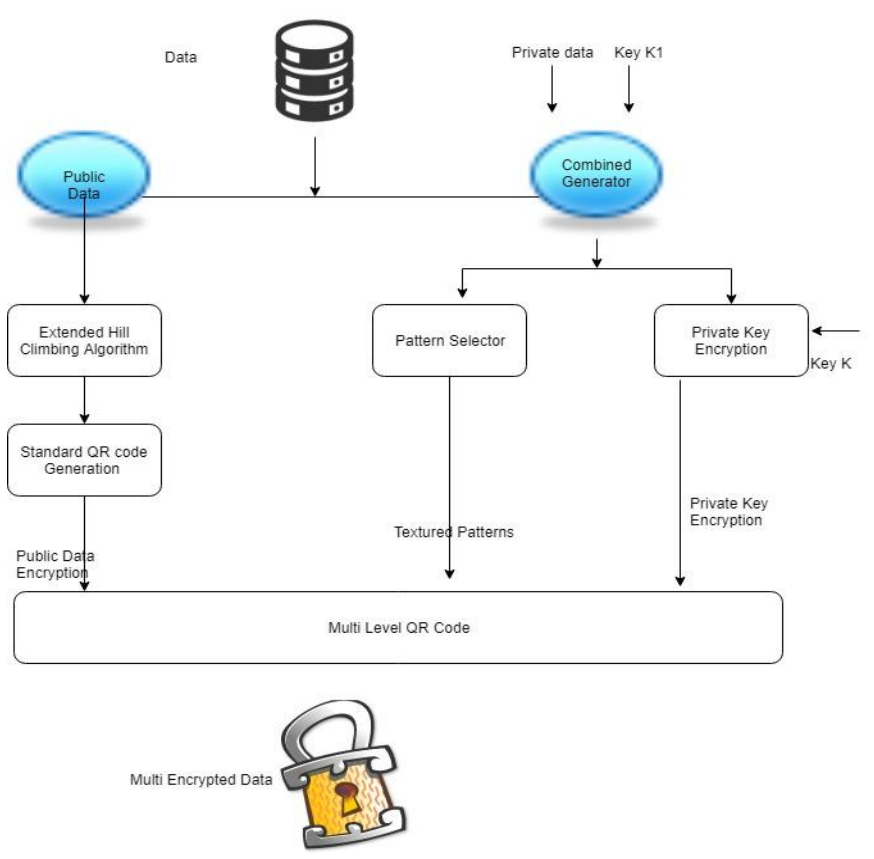

Fig 2: Multi QR code Generation methods

This will be prevailing by the choosing of the private key of the client decision and there is no limitation in choice of the key by the client and the key will be requested by the square lattice as same as the arranged by the open information and it will likewise be changed over into its ASCII esteem.

In this way the changed over open message will be duplicated with the key lattice dependent on the numbering the grid from left to right and in this way the resultant will be finished in like manner. The resultant network will be masterminded in the consecutive request and the processed framework will again be registered its ASCII esteems and the ASCII esteems will be included from the numbered resultant lattice from left to right.

Consequently the above figured information will be send to the open stockpiling level for transmission along these lines the standard scanner might probably peruse the scrambled registered information accordingly guaranteeing the security over the open information.

The private message(Mpriv) that should be scrambled will be first gets joined with the $\operatorname{key}(\mathrm{K} 1)$ which is utilized for encoding the open key is done at the join generator pursued by the determination of the example which is utilized for the information stockpiling and the private QR code scanner will be just ready to break down the example which will be chosen from the example database and the fulfillment of the protection is done uniquely with the encryption system subsequently the encryption of the information is being finished with the assistance of ECC encoding and the codeword of the information will be finished by the scrambling with the assistance of the outer private key(K2). Along these lines all the blend of the mixed code word with the chose finished examples and the standard QR code will be consolidated and the substitution of finished examples on the dark modules in this manner making proposed QR code clear just to the endorsed QR code scanner for the comprehensibility of the private information while all the standard QR code scanner could ready to peruse the open information anyway the open information will be encoded.

\section{B. Properties}

The proposed QR code has distinctive highlights with multi usefulness ascribes that adds to the attributes of recommended QR code. The significant element of QR code is the choice of finished example structure the example database where it contains set of the example from which the example must be chosen, the example ranges from the $\mathrm{Pi}, \mathrm{i}=1, \ldots, \mathrm{w}$ are being the pictures of size $\mathrm{p} \times \mathrm{p}$ pixels. Where $\mathrm{w}$ alludes to the greatest furthest reaches of the size of the information to be moved. The connection variables of the determination of the finished examples should be utilized, the relationship procedures can likewise be utilized with numerous examples. Hence relationship elements are to be determined.

The open message which should be sent is likewise being scrambled before putting away in the open stockpiling level additionally the private key which utilized for encode the open information will likewise be send by means of QR by blending the key with the private message and the joined data will be experience the private encryption systems and furthermore during the checking of the open message by the standard QR code scanner will ready to recover the encoded message as it were.

When the finished examples chose the subsequent stage is being trailed by the substitution of finished examples on the dark modules. Codeword C is included QR code which will begin with the base right corner. For mystery message situation, the finished example will be supplanted at the position zone concerning the chose stage $\sigma$ while validation idea, the position labels kept unaltered in dark modules.

In this way with the presentation of the finished examples the capacity properties in the QR code gets expanded drastically. This is predominantly because of the changing of the dark modules by the finished examples and the adaptation number is for the most part dependent on the capacity properties in the QR Thus the finished example fundamentally decides the capacity and the $\mathrm{QR}$ code rendition number therefore making the $\mathrm{QR}$ code unmistakable with the determination of the finished example there by making distinctive capacity limit just as the blunder revision limit. 


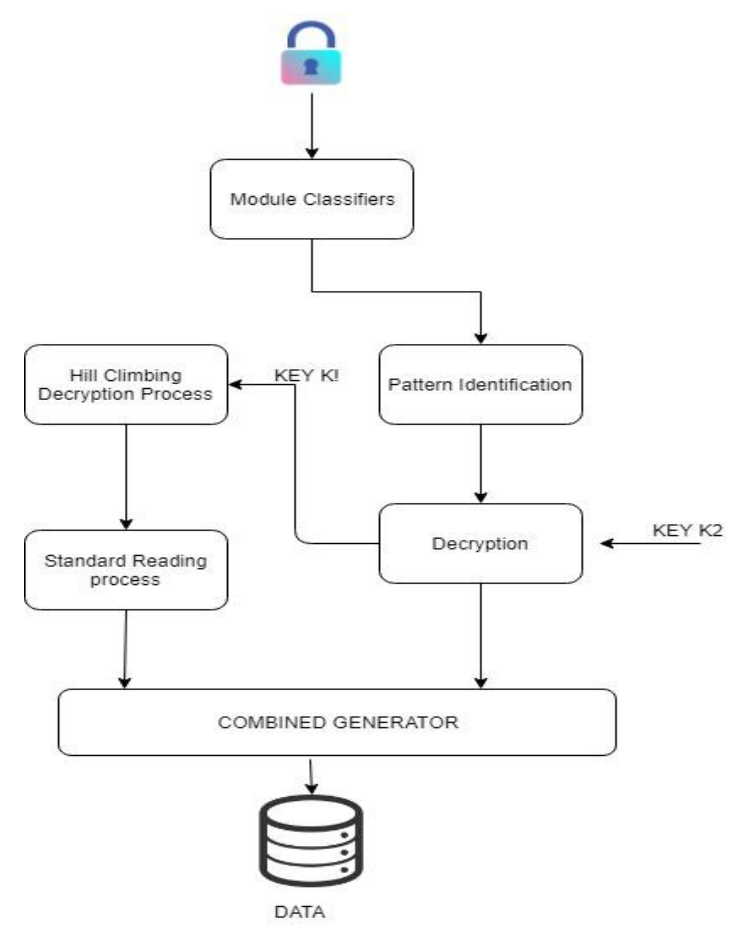

Fig 3: Multi QR code Decryption process

The acknowledgment of the proposed QR code is exceptionally a significant assignment in the process as it's a two level procedure as open information will examined by the QR code scanner anyway just the encoded substance of the open key will be shown while the private message can be perused distinctly by the endorsed QR code scanner where in which the finished examples database is accessible.

The diagram of the proposed QR code perusing procedure is being delineated is appeared underneath at first the $Q R$ code will experience the picture preprocessing strategy for the remaking of the QR code for the mistake redress system along these lines once this progression is finished the Reconstructed $\mathrm{QR}$ code is accessible this is trailed by the isolation of the information into the private and open information, Thus the open message will experience following procedure where in which at first the message will pursue the acknowledgment technique for the decoding slope figure therefore the message will be unscrambled now and hence now the standard QR code scanner can ready to peruse the information with the reasonable worth.

The above procedure will be portrayed as the key will be extricated dependent on the private message an incentive as the private message and the key utilized for the open key encryption strategy is additionally extricated here. In this manner the key used to decode the open message is accessible at this point.

Presently a similar procedure is relevant for the private message unscrambling system. Where the example acknowledgment is the main strategy to recognize the finished example pursued by the descrambling system where in which the key (K2) is utilized to unscramble the private message in this manner the information after this progression is the blend of the private information and the key (K1) ,the above advance is pursued be the de-mix method to isolate the $\mathrm{K} 1$ and the private message, The key will be continue to the open message level for the decoding procedure of the open message.

Along these lines the proposed QR code scanner could ready to peruse the private message and open message will be perused by the ordinary QR code scanner. Along these lines the information is accessible for the further procedure.

\section{EXPERIMENTAL ANALYSIS}

The proposed QR code will be presently experiencing arrangement of investigation so its effectiveness will be definite considered and talked about. Many test situations can be talked about out of which the capacity limit is examined beneath in the two test results.

At the last test we may raise the capacity size of proposed QR code by lessening the limit of the finished example in this manner keeping measurement consistent we would change the variant yet notwithstanding if the capacity limit is raised the mistake adjustment level and precision would diminish definitely and this would prompts the trustfulness of the information present in the QR code transmission with the blunder rate is expanding generally to the estimation of practically rising exponentially the outcome is appeared table I .

TABLE I.

THE STORAGE CAPACITY WITH RESPECT TO ALPHABET DIMENSION

\begin{tabular}{|l|l|l|l|}
\hline $\begin{array}{l}\text { Alphabet } \\
\text { Dimension }\end{array}$ & $\begin{array}{l}\text { Public } \\
\text { Level bit } \\
\text { data }\end{array}$ & $\begin{array}{l}\text { Private } \\
\text { Level bit } \\
\text { data }\end{array}$ & $\begin{array}{l}\text { Total } \\
\text { Number of } \\
\text { Data }\end{array}$ \\
\hline 6 & 272 & 208 & 480 \\
\hline 11 & 272 & 1168 & 1440 \\
\hline 18 & 272 & 2904 & 3176 \\
\hline
\end{tabular}

The subsequent experiment will also concentrates on the increasing of the storage this time the textured pattern is kept constant but the version number and the alphabet dimensions will be a variable one. This experiment also proves to be a valuable one but contrast to the first the error correction level is on an increasing side and thereby the error rate is also decreased dramatically. Thus the above experiments can be easily depicted in the table II.

TABLE II.

THE STORAGE CAPACITY WITH RESPECT TO TEXTURED PATTERN

\begin{tabular}{|l|l|l|l|l|}
\hline $\begin{array}{l}\text { Textured } \\
\text { Pattern }\end{array}$ & Version & $\begin{array}{l}\text { Public } \\
\text { Level bit } \\
\text { data }\end{array}$ & $\begin{array}{l}\text { Private } \\
\text { Level bit } \\
\text { data }\end{array}$ & $\begin{array}{l}\text { Total } \\
\text { Number } \\
\text { of Data }\end{array}$ \\
\hline $53 \times 53$ & 9 & 816 & 240 & 1,056 \\
\hline $81 \times 81$ & 16 & 1360 & 664 & 2,024 \\
\hline $121 \times 121$ & 26 & 1632 & 4400 & 6,032 \\
\hline
\end{tabular}

\section{CONCLUSION AND FUTURE WORK}

In this paper we propose moving the information with the assistance of QR code in 
the protected way with two degree of capacity level dependent on the significance of the information we classify the information and the data will be set in both of the capacity levels and both the capacity level data's will be encoded dependent on the distinctive encryption procedures chose dependent on their intricacy level, Thus open degree of data goes with the all-encompassing Hill climbing calculation while the private data goes with the ECC encryption calculation. Along these lines the information once encoded will be completely and totally perused by just by the chose QR code scanners with the example database detest in it. Consequently making the exchange of the data more verified too reliable. With the trial investigation we additionally demonstrated that the data moved is progressively exact with less number of mistake adjustment level required along these lines ensuring the data can have still more space for exchange.

In this manner the proposed idea can be still progressively adaptable in information limit with the blunder rectification levels.

\section{A. Future Work}

The QR code idea being a clear one numerous expansions should be possible concerning the setting going ahead with the proposed idea can be reached out by with including still more highlights like shading codes in the QR code or with the further developed encryption system substituting the current ones this can be additionally utilized in different fields like validness, Travel, Ticketing and if conceivable in IoT gadgets for transmission of the information. The age steps can likewise be made less difficult with consideration of the mechanization systems accordingly making the age and distinguishing proof procedure in an increasingly summed up way.

\section{REFERENCES}

1. Wu M, Liu B (2004) Data hiding in binary image for authentication and annotation. IEEE Trans Multimedia 6(4):528-538

2. Yang H, Kot AC (2007) Pattern-based data hiding for binary image authentication by connectivity-preserving. IEEE Trans Multimedia 9(3):475-486

3. Varna A, Rane S, Vetro A (2009) Data hiding in hard-copy text documents robust to print, scan and photocopy operations. In: Proc. of IEEE International Conference on Acoustics, Speech and Signal Processing, pp 1397-1400

4 Das S, Rane S, Vetro A Hiding information inside structured shapes. In: Proc. of IEEE International Conference on Acoustics Speech and Signal Processing, Mar 2010, pp 1782-1785

5. Kieseberg P, Leithner M, Mulazzani M, Munroe L, Schrittwieser S, Sinha M, Weippl E (2010) QR code security. In: Proc. of the International Conference on Advances in Mobile Computing and Multimedia, pp 430-435

6 Jing Q, Vasilakos AV, Wan J, Lu J, Qiu D (2014) Security of the internet of things: Perspectives and challenges. Wirel Netw 20(8):2481-2501

7 Reed IS, Solomon G (1960) Polynomial codes over certain finite fields. J Soc Ind Appl Math 8(2):300-304

8 Wilds M, Chambers S Bar code authentication, Patent US 2010/0 012 736 A1, Jan., 2010

9 Eldefrawy M, Alghathbar K, KhanMHardcopy document authentication based on public key encryption and 2D barcodes. In: Proc. of International Symposium on Biometrics and Security Technologies, Mar. 2012, pp 77-81

$10 \mathrm{Li} \mathrm{CM}, \mathrm{Hu}$ P, Lau WC Authpaper: Protecting paper-based documents and credentials using authenticated 2d barcodes. In: 2015 IEEE International Conference on Communications (ICC). IEEE, 2015, pp 7400-7406
11 Yang H, Kot AC (2007) Pattern-based data hiding for binary image authentication by connectivity-preserving. IEEE Trans Multimedia 9(3):475-486

12 Reed IS, Solomon G (1960) Polynomial codes over certain finite fields. J Soc Ind Appl Math 8(2):300-304

13 Villan R, Voloshynovskiy S, Koval O, Pun T (2006) Multilevel 2-D bar codes: Toward high-capacity storage modules for multimedia security and management. IEEE Trans Inf Forensics Secur 1(4):405-420.

\section{AUTHORS PROFILE}

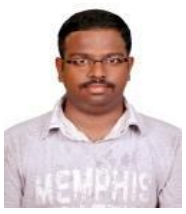

Gokul K is currently pursuing M.tech in cyber Security , National Institute Of Technology, Kurukshetra, India.

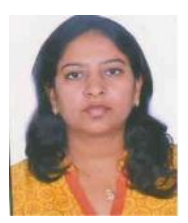

Dr. Ritu Garg is currently working as Assisstant Professor in National Institute of Technlogy,Kurukshetra,India 